\title{
Construção do Sistema Regional de Inovações do Estado do Amazonas a partir do Pólo Industrial de Manaus: histórico e resultados
}

\author{
Márcia Jucá Teixeira Diniz* \\ Marcelo Bentes Diniz** \\ Francisco Sérgio Silva Araújo***
}

\begin{abstract}
Resumo: Este artigo faz uma breve relação da dinâmica do Polo Industrial de Manaus (PIM) e alguns indicadores do estado do Amazonas, sob a ótica do Sistema Regional de Inovação. Faz-se uma rápida analise histórica do PIM desde a sua criação, além da análise de alguns de seus indicadores pelos dados da Superintendência da Zona Franca de Manaus (SUFRAMA). Em seguida, utilizam-se alguns indicadores do estado do Amazonas, fazendo referência também aos dados da Pesquisa de Inovação Tecnológica - PINTEC/IBGE e às informações do Diretório dos Grupos de Pesquisa do CNPq de 2010. Dessa forma, como resultado, evidencia-se um processo em construção de um Sistema Regional/Local de Inovação no estado do Amazonas, podendo ser considerado como um "ponto de interação" na região Norte e mesmo no cenário brasileiro.
\end{abstract}

Palavras-Chave: Sistema Regional de Inovação, Polo Industrial de Manaus, Amazonas.

Classificação JEL: R12.

\footnotetext{
*Doutora em Desenvolvimento e Meio Ambiente - NAEA-UFPA. Professora do Programa de Pós-Graduação em Economia e Faculdade de Ciências Econômicas da Universidade Federal do Pará. E-mail: marciadz2012@, hotmail.com

**Doutor em Economia - CAEN-UFC. Professor do Programa de Pós-Graduação em Economia e Faculdade de Ciências Econômicas da Universidade Federal do Pará. E-mail: mbdiniz2007@hotmail.com

*** Graduado em Ciências Econômicas - UFPA. Aluno de Mestrado do Programa de Pós-Graduação em Economia da Universidade Federal do Pará. E-mail: nfg1985@bol.com.br
} 


\section{Introdução}

O Polo Industrial de Manaus pode ser considerado um exemplo típico da interação entre empresas, universidade/instituições de pesquisa e governo (Hélice Tripla), conformando-se como um produto do Sistema Regional/Local de Inovação, no estado do Amazonas. Esse sistema, amplamente discutido na literatura (Nelson e Rosenberg, 1993; Freeman, 1995 e Lundvall, 2003), ébaseado na capacidade de inovação por parte das empresas que têm como suporte a ação de vários agentes econômicos - governo, institutos de pesquisa e universidade orientadas a esse intento (Cohen e Levinthal, 1990). Em verdade, atualmente, é grande a importância dada ao processo inovativo que ocorre na empresa e às relações que se estabelecem entre ela e seu entorno como determinante da competitividade dos países (Dagnino, 2003).

Se a ação de aglomerados industriais assume o formato de um Cluster, Distrito Industrial, Rede de Firmas, Complexo ou Pólo Industrial varia em função do grau de interação, sinergia, complexidade das relações estabelecidas e resultados recíprocos recebidos entre as empresas envolvidas. Nesse particular, segundo Souza (2005,p.88), "um complexo industrial é um conjunto de atividades ligadas por relações de insumo-produto. Ele forma um polo de crescimento quando for liderado por uma ou mais indústrias motrizes; e ele se tornará um polo de desenvolvimento quando provocar transformações estruturais e expandir o produto e o emprego...".

Dessa forma, cabe, neste artigo, a seguinte indagação: um aglomerado de empresas que se conformam como um Polo Industrial, geograficamente localizado dentro de uma região tão vulnerável como a região Amazônica, e mais especificamente, no estado do Amazonas, é capaz, via processos contínuos de inovação das suas empresas, tanto para o mercado local, como nacional e até externo, trazer consequências positivas à formação de um Sistema Regional/ Local de Inovação com efeitos para o desenvolvimento desse estado?

Para responder tal questionamento, faz-se necessário então discutir alguns pontos. O primeiro refere-se à definição de sistemas nacionais/regionais ou mesmo locais de inovação, discutida na próxima sessão. O outro ponto referese à criação do polo industrial de Manaus e as fases pelo qual passou, até sua consolidação nos dias atuais, relatado na sessão três. Em seguida, na sessão quatro, elucida-se alguns indicadores socioeconômicos do estado do Amazonas, mostrando a forte relação com o PIM, utilizando-se também dos dados da PINTEC/IBGE. Por fim as considerações finais.

\section{Sistema Nacional/Regional de Inovação}

O Sistema Nacional de Inovação e, mesmo, os Sistemas Regionais (e/ou estaduais) de Inovação se caracterizam pela interação das ações institucionais, intencionais ou não, que impulsionam o progresso tecnológico e, dessa forma, integram tanto a atividade de inovação propriamente dita, como a 
cooperação entre firmas e instituições geradoras e difusoras de conhecimento e desenvolvimento científico e tecnológico, que atuam em uma determinada área geográfica, espacialmente definida ${ }^{1}$ (Lundvall, 1992; Nelson e Rosenberg, 1993; Freeman, 1995; Lundvall, 2003; Edquist, 2005; Doloreux e Hommen, 2003).

Ressalta-se que através da construção desse sistema de inovação viabiliza-se a realização de fluxos de informação necessários ao processo de inovação tecnológica (Nelson e Rosenberg, 1993), no qual integram e interagem, nesse processo, instituições formais que produzem, difundem o conhecimento, como as empresas e as organizações de ensino e pesquisa, mas também outros atores e instituições, inclusive políticas, que afetam o desenvolvimento desses sistemas (Cassiolato e Lastres, 2008).

Ademais, sob o entendimento do funcionamento sistêmico do SNI, são importantes os fluxos de interação, complementaridades e sinergias entre os diversos atores que compõem seu funcionamento, direta e indiretamente relacionado aos mecanismos/fontes de inovação, as redes sociais e o ambiente interno no país, como as condições macroeconômicas, políticas, sociais, institucionais, financeiras de cada economia. E como acentua Fagerberg (2005), esse sistema dinâmico tem seus feedbacks, que podem reforçar ou enfraquecer a estrutura e funcionamento do mesmo, levando a possíveis configurações de "lock in" ou "lock out".

De fato, como explicita Suzigan et al. (2011), no Sistema Nacional de Inovação é importante a existência de "circuitos retroalimentadores" entre duas importantes dimensões - científicas e tecnológicas - podendo ser consideradas positivas quando os fluxos de informação e conhecimento se dão em dois sentidos, que sejam, as universidades e institutos de pesquisa produzem conhecimento científico que, por sua vez, é absorvido pelas empresas, e estas acumulam conhecimento tecnológico, fornecendo questões para a elaboração científica.

A abordagem dos sistemas de inovação coloca o processo de aprendizado e inovação como foco central, e que tem metodologicamente cinco aspectos fundamentais (Edquist, 2005): i) uma perspectiva holística e interdisciplinar; ii) emprega uma visão histórica e evolucionária, o qual faz a noção de optimalidade irrelevante; iii) enfatiza a interdependência e a não-lineariedade; iv) encampa tanto inovações em processos, como inovações de produto, tanto quanto subcategorias das mesmas; v) enfatiza o papel das instituições.

Mais especificamente, a contribuição da pesquisa acadêmica para o avanço tecnológico ocorre por vários mecanismos, além de variar entre os setores industriais e de existirem ramos da ciência cujos avanços são considerados mais relevantes para as inovações tecnológicas. Klevorick (1995) aponta os avanços no conhecimento científico como sendo a fonte mais importante de oportunidades tecnológicas. No entanto, em países em desenvolvimento, devido a uma estrutura industrial que investe pouco em P\&D, e com pouca absorção de mão de obra qualificada, o compartilhamento do conhecimento produzido nas universidades e institutos de pesquisa pode ser comprometido - as universidades produzem

1 A base de um sistema de inovação é construída através dos setores produtivos e as empresas que "saem na frente" (em termos de inovação, como uma típica empresa schumpeteriana), incorporam continuamente conhecimento tácito, estratégias direcionada à inovação, Know how, baseada em atividades de rotina (Nelson;Winter, 1982). 
ciência para dar suporte à atividade inovativa das empresas (Klevorick, 1995; Cohen, 2002).

O conceito mais estrito de Sistema Regional de Inovação retrata essa mesma perspectiva, só que entendendo que no âmbito do sistema maior, nacional, possam coexistir subsistemas como uma delimitação espacial de interligação dos agentes econômicos em suas diversas interfaces. Esses subsistemas existem e reproduzem, em geral, as desigualdades históricas, quanto às assimetrias econômicas e sociais entre as economias subregionais.

Por outro lado, a abordagem de Sistema Nacional/Regional de Inovação rompe com alguns conceitos sobre inovação, deixando de ser considerada apenas como processo de mudança radical, em setores de tecnologia de ponta, realizadas por grandes empresas. Mas, como assinalam Mytelka e Farinelli (2005), compreende atividades realizadas, também, por pequenas e médias empresas e o reconhecimento de que a inovação se estende para além das atividades formais de P\&D.

Ainda por essa abordagem, entende-se que a inovação e o desenvolvimento econômico originam-se de condições particulares, sociais, organizacionais, institucionais e de características histórico-culturais. São os elementos e as relações presentes em determinado sistema que poderão determinar a capacidade de aprendizado e inovação de um país, região ou localidade. E, por tudo isso, o SNI e SRI têm uma importância decisiva no processo de desenvolvimento dos países (Gordon, 2008), tanto em escala macronacional, como na escala macroregional, inclusive, quanto as suas assimetrias internas.

No contexto do Sistema ou como um produto do mesmo a interação entre empresas, universidades/instituições de pesquisa e governo tem um papel decisivo na dinâmica dos investimentos dos setores produtivos a partir da própria dinâmica da produção científica e de estratégias de proteção desse conhecimento (Cassiolato, 2010), incorporado ao processo produtivo "enquanto um negócio baseado em ciência".

Em verdade, a cooperação entre os três agentes citado acima, conformam a base do argumento da Hélice Tripla, modelo sistematizado pelos trabalhos de Etzkowitz e Leydesdorff (1998). Esse modelo objetiva ultrapassar os limites impostos pela visão linear do processo de inovação (Brisolla et al, 1997), através da interação entre universidades, empresas e o Estado, com intuito de promover o desenvolvimento econômico (Inzelt, 2004; Farinha, Ferreira, 2013)

Nesse sentido, o modelo proposto por Farinha e Ferreira (2013) dá a noção exata das relações entre empresa, universidade/institutos de pesquisa e governo. Pela figura 1 abaixo, os autores acima propõem uma "triangulação da hélice tripla" como modo de avaliar os tres pilares para a competitividade e desenvolvimento regional, onde a inovação e o empreendedorismo atuam como fatores essenciais para esse fim, pois estimulam novos investimentos, criam emprego e renda, impulsionando, assim, as economias a atingirem novos padrões de competição regional. 


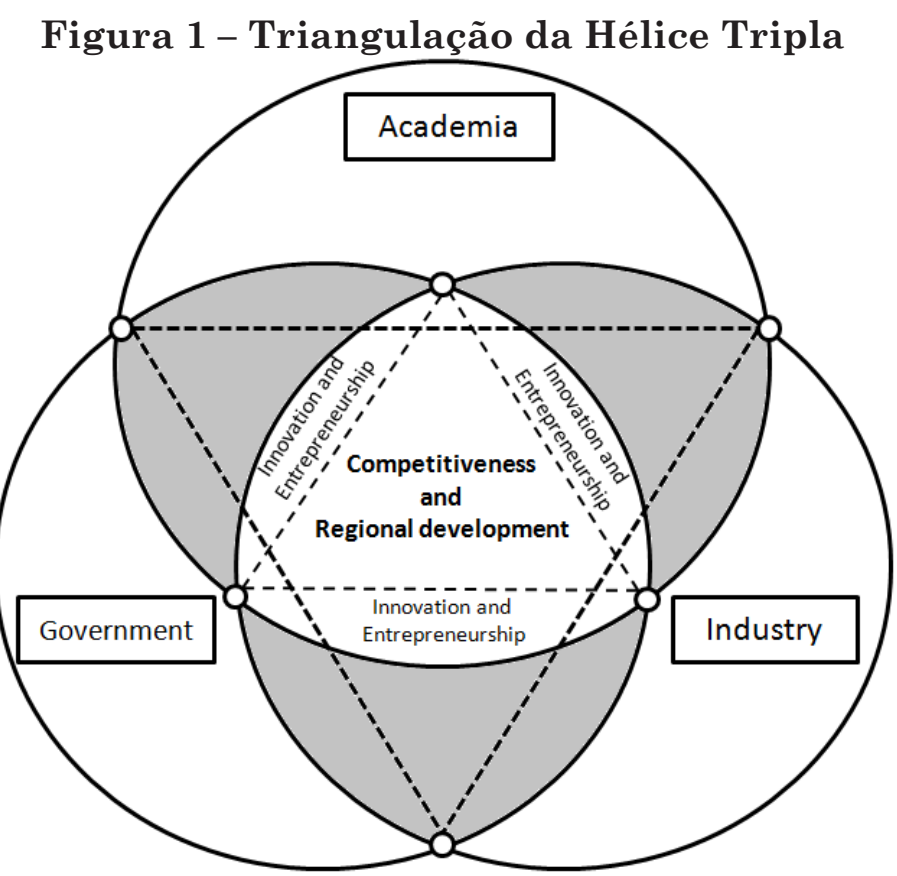

Fonte: Farinha;Ferreira (2013)

Por essa via, Cooke (2001) elabora e explicita as condições de uma determinada economia (local ou regional) construir um maior ou menor poder econômico dentro da abordagem do Sistema Regional de Inovação. Elas estão sumarizadas no quadro 1 abaixo.

\section{Quadro 1 - Sistema Regional de Inovação-Condições para a Eficiência e Ineficiência}

\begin{tabular}{ll}
\hline \multicolumn{1}{c}{ SRI - Potencialmente Eficiente } & \multicolumn{1}{c}{ SRI - Potencialmente Ineficiente } \\
\hline 1) Nível Geral de Infraestrutura: & 1) Nível Geral de Infraestrutura: \\
- Autonomia Tributária e de Gastos & - Gastos Descentralizados \\
- Financiamento Privado Regional & - Organização Financeira Nacional \\
- Influencia Política em Infraestrutura & - Influencia Limitada sobre a Infraestrutura \\
• Estratégia Regional na relação & - Projetos de Inovação Fragmentários \\
Universidade-empresa & - Culturas Competitivas, Individualismo e \\
• Cultura Cooperativa, Aprendizado & Divergências Institucionais \\
Interativo e Consenso Associativo & 2) Dimensão Organizacional - Firmas: \\
2) Dimensão Organizacional - Firmas: & - Relações de Trabalho antagônicas \\
- Relações de Trabalho Harmoniosas & - Habilidades Auto-adquiridas \\
- Trabalhador orientado(qualificado) & - Internalização \\
- Externalização & - P\&D Autônomo \\
- Inovação Interativa - criação de spinoffs & 3) Dimensão Organizacional - Política: \\
3) Dimensão Organizacional - Política: & - Exclusiva \\
- Inclusão & - Reativa \\
- Monitoramento & - Autoritária \\
- Consultivo & - Hierárquica \\
\hline Redes & Fonte: Modificado de Cooke (2001) - Elaboracaão dos autores
\end{tabular}

Fonte: Modificado de Cooke (2001) - Elaboração dos autores 
Assim, em relação ao quadro acima, verifica-se o antagonismo de quase todas as condições quanto a um eficiente e ineficiente SRI. Segundo Cooke (2001), a internalização das atividades relacionadas à dimensão organizacional das firmas conduz, no ineficiente SRI, apenas à adaptação de inovações oriundas de fora da empresa. Conquanto, para o SRI eficiente, a dimensão relacionada à política (ações políticas) se compõe de inclusão (social e econômica), monitoramento, consultoria e redes de cooperação entre os policymakers. Em verdade, são regiões diferenciadas pela própria integração de todos os agentes sociais ou não.

Uma estratégia de desenvolvimento (sistêmica), baseada no SRI, tem como ponto de partida, uma análise de todos os setores da economia que contribuem para o desenvolvimento de competências visando à inovação. Essa análise pode identificar as redes de estímulo ao aprendizado e as redes e interações ausentes. Em verdade, o Sistema Regional de Inovação tem como pressuposto uma interação acentuada entre a ciência pura (Rosenberg, 2006) e a tecnologia, mostrando que as empresas obtêm melhores resultados à medida que a pesquisa básica progride.

Por sua vez, Suzigan e Albuquerque (2006) destacam as principais contribuições das instituições cientificas em um Sistema Regional de Inovação imaturo ou ineficiente: instrumento de focalização contribuindo para a identificação de oportunidades e de vinculação do país aos fluxos internacionais; apoio para o desenvolvimento industrial, fornecendo o conhecimento necessário para a entrada em setores industriais estratégicos; e, como fonte para algumas soluções criativas que dificilmente seriam obtidas fora do país.

Segundo Cário et al., (2011), os estados da região Norte possuem poucas instituições de ensino superior e a interação entre essas poucas instituições de ensino e também de pesquisa com as empresas é bem menor, especialmente se levadas em conta outras regiões do país. Em verdade, o autor acima considera que tais interações estão em processo de construção/constituição, em que as bases para essas interações positivas ainda não estão constituídas.

Em verdade, especialmente, no Brasil, deve-se levar em conta as diferenças inter-regionais quanto à distribuição de indicadores de Ciência, Tecnologia e Inovação (Costa, 1998; Becker, 2006; CCGE, 2009), ou mesmo da Economia do Conhecimento como evidenciam Diniz e Gonçalves (2005), que acabam por reforçar a lógica de exploração das empresas de caráter nacional e os "produtores locais", ao mesmo tempo, que não se criam elementos endógenos para "quebrar" esse processo.

É sobre esse prisma que aparece uma dicotomia muito grande quando se compara os indicadores de Ciência, Tecnologia e Inovação, dos estados da região norte (Amazônia), em comparação às regiões mais desenvolvidas do país, sudeste e sul e, mais ainda, com relação à média do desempenho mundial, com reflexos na conformação e amadurecimento da tríplice hélice na região e, por sua vez em um Sistema Regional de Inovação que dê conta das exigências que o setor produtivo das atividades econômicas impõe para seu funcionamento e competitividade. 
De forma geral, as condições para um SRI eficiente ou ineficiente (imaturo), constatadas no quadro acima, podem ser analisadas à luz de vários indicadores como: interação entre universidades e empresas (grupos de pesquisa), gastos estaduais e federais em ciência e tecnologia, níveis salariais da mão de obra empregada nas empresas, qualificação da mão de obra, nível de investimento em P\&D, e tantos outros que poderiam servir para uma conclusão objetiva sobre um SRI na Amazônia

\section{O Pólo Industrial De Manaus - PIM e a conformação do SRI}

A Zona Franca de Manaus, através de seus incentivos, possibilita, desde muito tempo, a implantação de projetos via Superintendência da Zona Franca de Manaus - SUFRAMA - para instalação de um parque industrial mais diversificado na região (Cano, 1997; Martinello, 1988). Ela foi instituída em 1957 com a Lei no. 3.173, e regulamentada em fevereiro de 1960, e, de acordo, à época, com a política nacional, optou-se por fazer completa reestruturação nos incentivos então vigentes para a Amazônia brasileira. Entre eles: a transformação do Banco de Credito da Amazônia S.A. em Banco da Amazônia S. A. - BASA; a criação da SUDAM; e a concessão de incentivos fiscais em favor da região.

Essas mudanças culminaram com o Decreto-Lei n. 288 de fevereiro de 1967 onde fixava em 30 (trinta) anos o prazo de vigência dos incentivos fiscais - admitindo sua prorrogação. Desde a sua promulgação, o Decreto-Lei passou por inúmeras mudanças em especial, relacionadas à isenção de impostos e aos impostos referentes ao destino dos produtos ali produzidos.

De forma mais objetiva, pode-se entender a evolução histórica das indústrias incentivadas de Manaus - Polo Industrial de Manaus (PIM) - e, portanto, sua dinâmica até os dias atuais, através da periodização feita por Machado et al. em 2006 e pela Superintendência da Zona Franca de Manaus - SUFRAMA (2013). Assim, o primeiro período vai da implantação do DecretoLei n. 288 em 1967 até meados da década de 1970, mais especificamente, 1976. Essa é a fase de criação de mercado interno para bens finais inéditos pela atração de empresas internacionais, majoritariamente em eletrônica de consumo, com liberdade de importação de insumos. Sendo assim, constituiram-se as etapas iniciais na formação de mercado interno de bens de consumo duráveis nesse segmento, inclusive possibilitando a entrada no Brasil de tecnologia só disponível nos países desenvolvidos.

O segundo período do PIM - fim da década de 1970 até início dos anos de 1990 - a lógica de suporte do PIM, nessa fase, foi a substituição de importações calcada no privilégio a projetos produtivos que contemplassem índices de nacionalização elevados na aquisição de insumos. A necessidade de estimular a verticalização de alguns setores industriais era iminente, além de investir na formação de capacitação técnica e na geração de conhecimento cientifico e 
tecnológico, o que foi realizado através de convênios entre a Superintendência da Zona Franca de Manaus - SUFRAMA - e instituições de ensino, pesquisa e desenvolvimento, repassando-lhes recursos para execução de projetos, os mais diversos (Machado et al. 2006).

Entre os anos de 1990 e 1996, a chamada $3^{\text {a }}$ etapa (ou terceiro período) do Pólo Industrial de Manaus, inserido no Modelo ZFM, está ligada à fase de modernização industrial high-tech, num ambiente, agora, globalizado. As indústrias incentivadas de Manaus sentiram as conseqüências da abertura comercial brasileira (importações em quantidades livres) aliadas à política de estabilização monetária do período de 1993/1994. Assim, sofrendo as consequências da competição externa, as indústrias incentivadas de Manaus viram-se obrigadas a alterar substancialmente sua função de produção, passando a ser mais intensivas em capital e tecnologia, no qual passou a promover um esforço de modernização, cujas velocidades e profundidade dificilmente possuem paralelo na história industrial brasileira. Nos primeiros anos de reestruturação do $\mathrm{PIM}^{2}$, a quantidade de mão-de-obra direta foi bastante reduzida, mas a qualidade e as condições de empregos restantes melhoraram substancialmente, tendo legítimos ganhos de produtividade (Machado et al., 2006; Garcia, 2004).

O quarto período elucidado pela Suframa (2013), compreende o período de 1996 a 2002, em que a política industrial de referência do país caracterizavase por sua adaptação aos cenários de uma economia globalizada e pelos ajustes demandados pelos efeitos do Plano Real, como o movimento de privatizações e desregulamentação.

No período atual (fase atual), entra em vigor a Política de Desenvolvimento Produtivo (PDP), em consonância com a Política Industrial Tecnológica e de Comércio Exterior (PITCE), objetivando maior eficiência produtiva e capacidade de inovação das empresas e expansão das exportações (SUFRAMA, 2013).

Em verdade, os incentivos dados às indústrias do PIM estão vinculados aos tributos incidentes sobre o valor agregado nas atividades de industrialização e comercialização - IPI e ICMS - e aos tributos aduaneiros - IE e II- incidentes sobre a importação de insumos estrangeiros e a exportação de bens produzidos na região. Esses incentivos são direcionados à produção e não ao capital, pois as empresas só usufruem deles quando produzem e vendem, não ocorrendo desembolsos do Erário federal e estadual para sustentar os projetos incentivados ( SUFRAMA, 2013; Grosso, 2005).

No novo cenário, a Suframa implantou uma política que integrasse três grandes vetores de ações essenciais (Machado et al. 2006): a) o adensamento das cadeias produtivas dos bens fabricados com incentivos em Manaus por meio de atividades de atração de novos produtores. Aliado a isso, os pesados esforços de promoção e investimento em Ciência, Tecnologia e Inovação (C,T\&I), capitaneados pela Suframa a partir de 1999, agora inseridos como item regular de sua agenda institucional, e realizados em parceria com instituições de pesquisa locais, públicas e privadas, no sentido de formar um sólido sistema

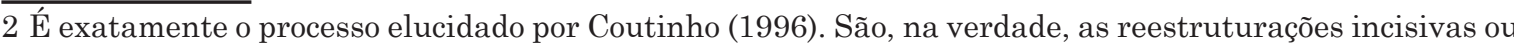
brutais das empresas (com profundos cortes de pessoal, custos fixos e de administração ou racionalização de processos produtivos), e seus respectivos resultados como ganhos de produtividade; significativas economias de capital de giro; estruturas administrativas e organizacionais enxutas e processos gerenciais mais eficientes. 
de C,T\&I que suportasse a competitividade da produção empresarial high-tech e que explorasse fronteiras de inovação; b) A internacionalização da indústria local - adotando mecanismos de estímulos às exportações e de programas de promoção comercial e de cooperação internacional; c) A interiorização dos resultados do desenvolvimento na Amazônia Ocidental - por meio de aproveitamento sustentável dos recursos naturais amazônicos e da aplicação dos recursos próprios da Suframa, gerados a partir da arrecadação de sua Taxa de Serviços Administrativos (TSA) em infraestrutura socioeconômica de apoio a projetos produtivos de cunho empresarial ou cooperativo.

O Pólo Industrial de Manaus, capitaneado pela SUFRAMA, sempre entendeu a necessidade de estimular a verticalização de alguns setores industriais, além de investir na formação de capacitação técnica e na geração de conhecimento cientifico e tecnológico, o que foi realizado através de convênios entre a SUFRAMA e instituições de ensino, pesquisa e desenvolvimento, repassando-lhes recursos para execução de projetos, os mais diversos. Projetos esses, voltados a estudos de ecologia florestal, produção de alimentos e, especialmente, a formação de recursos humanos. Entre essas instituições estavam: Instituto Nacional de Pesquisas da Amazônia - INPA, a Empresa Brasileira de Pesquisa Agropecuária - EMBRAPA, Empresa de Assistência Técnica e Extensão Rural - EMATER/AM, Fundação Universidade do Amazonas (MACHADO, 2006). Em suma, como citado acima, os avanços no conhecimento científico são a fonte mais importante de oportunidades tecnológicas (Klevorick, 1995) e, assim, um suporte para o aumento do poder concorrencial das empresas no mercado nacional e internacional.

Segundo Garcia (2004), ao criar a Zona Franca de Manaus, o governo Federal decidiu trocar receita tributária por resultados econômicos e sociais, gerando meios para impor competitividade à economia regional; diminuir as disparidades inter e intra-regionais e, estimular o desenvolvimento.

Em verdade, os resultados econômicos e sociais da renúncia fiscal, no polo industrial, são traduzidos em elevados estoques de investimento fixo e tecnologia de ponta e níveis significativos de produção, faturamento, renda e salário geram um efeito multiplicador bastante favorável sobre o desempenho da economia da região e consequentemente da economia nacional, determinando o crescimento da arrecadação de tributos federais e devolvendo à União parte dos meios por elas concedidos (Machado, 2006; Garcia, 2004).

\subsection{Dados Recentes sobre o Pólo Industrial de Manaus}

Atualmente, os incentivos fiscais podem ser resumidos da seguinte forma: 1) Federais - Redução de até 88\% do Imposto de Importação (I.I.) sobre os insumos destinados à industrialização; isenção do Imposto sobre Produtos Industrializados (I.P.I.); redução de 75\% do Imposto de Renda de Pessoa Jurídica, inclusive adicionais de empreendimentos classificados como prioritários para o desenvolvimento regional, calculados com base no Lucro da Exploração até 
2013; e, Isenção da contribuição para o PIS/PASEP e Cofins nas operações internas na Zona Franca de Manaus. 2) Estadual - Restituição parcial ou total, variando de $55 \%$ a 100\% - dependendo do projeto - do Imposto sobre Operações Relativas à Circulação de Mercadorias e sobre Prestação de Serviços de Transporte Interestadual e Intermunicipal e de Comunicação(ICMS). Em todos os casos as empresas são obrigadas a contribuir para fundos de financiamento ao ensino superior, turismo, $\mathrm{P} \& D$ e às pequenas e microempresas. 3) Municipais - Isenção do Imposto sobre a Propriedade Predial e Territorial urbana, Taxas de Serviços de Coleta de Lixo, de Limpeza Pública, de Conservação de Vias e Logradouros Públicos e Taxas de Licença para empresas que gerarem um mínimo de quinhentos empregos, de forma direta, no início de sua atividade, mantendo este número durante o gozo do benefício. (Lei Municipal nº 427/1998) (SUFRAMA, 2013)

O PIM, atualmente, na qualidade de modelo de produção regional formado por indústrias high-tech, apresentou, nos anos recentes, desempenho expressivo, não só como decorrência da dinâmica empresarial e de mercado em si, mas, também, como resultados dos três vetores estratégicos de ação referidos acima (Machado, 2006).

A dinâmica imposta pelo PIM ao Estado do Amazonas e, mais especificamente, ao município de Manaus, é atribuída à grande representatividade da arrecadação tributária, que em termos federais posiciona o Estado do Amazonas como o primeiro da Região Norte (Rivas et. al. 2008).

A tabela 1 mostra o grande montante de recursos investidos no PIM. Caracterizado por setores de atividades, os maiores investimentos estão nos setores eletroeletrônico, duas rodas e químico.

Tabela 1 - Investimentos Realizados no PIM por Setores - 2007-2013 U\$S1,00

\begin{tabular}{l|c|c|c|c|c|c|c}
\hline \multirow{2}{*}{ Setores } & \multicolumn{7}{|c}{ Anos } \\
\cline { 2 - 8 } & 2007 & 2008 & 2009 & 2010 & 2011 & 2012 & $2013\left(^{*}\right)$ \\
\hline $\begin{array}{l}\text { Eletro- } \\
\text { eletrônico }\end{array}$ & 2.479 .442 .912 & 2.692 .800 .323 & 2.509 .414 .493 & 2.901 .204 .824 & 3.368 .795 .308 & 3.294 .834 .161 & 3.755 .260 .631 \\
\hline Duas Rodas & 1.270 .580 .924 & 1.741 .425 .565 & 1.999 .685 .718 & 2.369 .474 .189 & 2.693 .237 .065 & 2.491 .393 .688 & 2.583 .204 .658 \\
\hline $\begin{array}{l}\text { Termo- } \\
\text { plástico }\end{array}$ & 749.174 .789 & 732.143 .629 & 735.692 .213 & 960.676 .831 & 986.286 .957 & 788.668 .842 & 831.159 .028 \\
\hline Metalúrgico & 304.566 .862 & 377.657 .472 & 416.461 .463 & 482.886 .826 & 565.404 .912 & 565.734 .755 & 568.654 .932 \\
\hline Mecânico & 352.537 .954 & 420.598 .043 & 382.748 .326 & 457.196 .765 & 593.467 .004 & 522.527 .577 & 562.636 .132 \\
\hline Químico & 513.415 .655 & 661.891 .960 & 618.986 .224 & 724.918 .168 & 814.042 .210 & 839.353 .273 & 860.209 .282 \\
\hline $\begin{array}{l}\text { Mineral não } \\
\text { Metálico }\end{array}$ & 208.235 .891 & 225.883 .215 & 211.014 .781 & 242.960 .842 & 257.376 .166 & 223.814 .019 & 214.675 .146 \\
\hline $\begin{array}{l}\text { Isqueiros, } \\
\text { Canestas e } \\
\text { outros }\end{array}$ & 366.539 .373 & 549.552 .647 & 527.940 .886 & 608.582 .644 & 704.543 .809 & 713.605 .862 & 777.470 .474 \\
\hline Outros & 458.612 .656 & 513.071 .857 & 493.975 .398 & 530.953 .060 & 669.579 .575 & 631.186 .082 & 608.329 .853 \\
\hline Totais & 6.703 .107 .016 & 7.915 .024 .711 & 7.895 .919 .502 & 9.278 .854 .149 & 10.652 .733 .006 & 10.071 .118 .259 & 10.761 .600 .136 \\
\hline
\end{tabular}

Fonte: COISE/CGPRO/SAP. SUFRAMA (2013)

$\left.{ }^{*}\right)$ Dados Parciais - Fevereiro 2013. 
No que se refere ao faturamento e receita total do PIM, a tabela 2 revela, desde 2003 (com exceção de 2009), um crescimento desses indicadores em todo o período, por parte das empresas nele localizadas.

Tabela 2 - Faturamento e Receita Total do PIM - 2000 - 2012 U\$S

\begin{tabular}{l|c|c|}
\hline Ano & \multicolumn{1}{c}{ Faturamento } & Receita Total \\
\hline $\mathbf{2 0 0 0}$ & 10.395 .099 .859 & 11.843 .133 .280 \\
\hline $\mathbf{2 0 0 1}$ & 5.543 .405 .108 & 10.218 .133 .666 \\
\hline $\mathbf{2 0 0 2}$ & 9.112 .939 .186 & 10.009 .556 .985 \\
\hline $\mathbf{2 0 0 3}$ & 10.622 .444 .765 & 11.186 .457 .419 \\
\hline $\mathbf{2 0 0 4}$ & 14.190 .897 .750 & 14.948 .279 .041 \\
\hline $\mathbf{2 0 0 5}$ & 18.901 .682 .280 & 20.008 .721 .304 \\
\hline $\mathbf{2 0 0 6}$ & 22.748 .004 .704 & 24.037 .945 .955 \\
\hline $\mathbf{2 0 0 7}$ & 25.669 .856 .080 & 28.929 .931 .955 \\
\hline $\mathbf{2 0 0 8}$ & 30.100 .335 .633 & 33.628 .128 .650 \\
\hline $\mathbf{2 0 0 9}$ & 25.953 .651 .605 & 28.448 .161 .189 \\
\hline $\mathbf{2 0 1 0}$ & 35.215 .281 .470 & 38.914 .412 .675 \\
\hline $\mathbf{2 0 1 1}$ & 41.237 .312 .265 & 44.108 .733 .126 \\
\hline $\mathbf{2 0 1 2}$ & 37.549 .642 .823 & 40.065 .239 .221 \\
\hline \multicolumn{2}{|c|}{ Fonte:SUFRAMA(2013) }
\end{tabular}

A Balança Comercial do PIM (Gráfico 1) é historicamente deficitária, apesar de as exportações se mostrarem crescentes em todo o período considerado -2007 a 2012.

Esse déficit ocorre, basicamente, por dois motivos: o primeiro está em função de que boa parte da produção, dos vários setores considerados, é destinada tanto ao mercado local como ao nacional, atestando, desde essa época, a esperada integração das empresas locais no polo. $\mathrm{O}$ segundo se deve exatamente a importação de produtos básicos/insumos necessários à produção de várias empresas do setor Eletroeletrônico e Duas rodas, como por exemplo - Samsung, Honda, LG e Nokia (SEPLAN, 2013). Ainda, essas empresas são intensivas em tecnologia especializada (de fronteira) onde a existência de similares nacionais é deficitária.

É nítida a evolução da mão-de-obra no período considerado, passando de 98.720 em 2007 para 120.056 em 2013 (SUFRAMA 2013). Ademais, como pode ser observado no gráfico 2 , este volume do emprego cresceu basicamente entre as faixas de - até 1,5 SM e 4,0 a 6,0 SM. No que se refere às mais altas faixas, houve uma pequena variação nas quantidades de pessoas empregadas e até mesmo uma redução na faixa mais alta - acima de 15,0SM (Gráfico 2) 


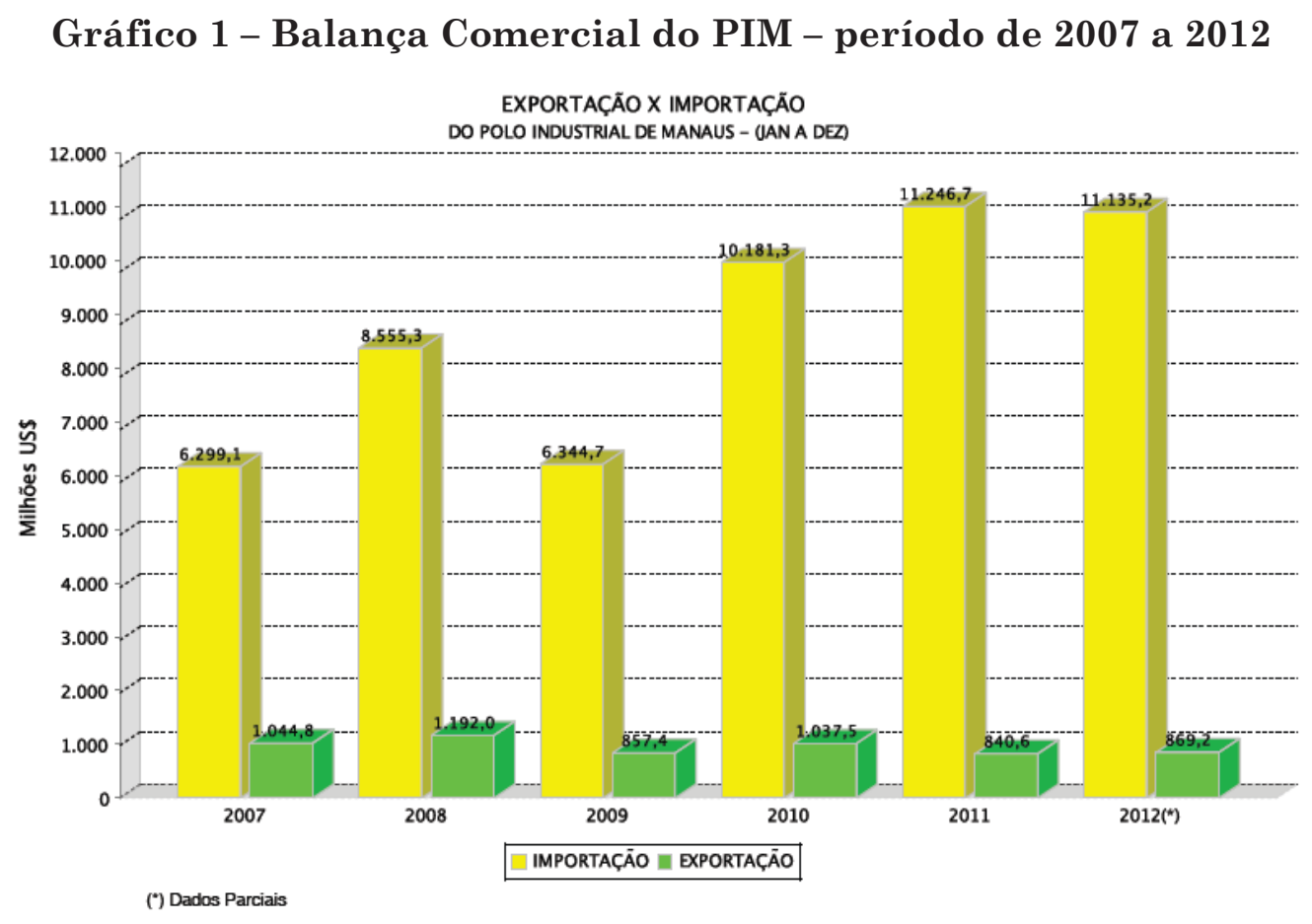

Fonte: COISE/CGPRO/SAP . Suframa (2013)

\section{Gráfico 2 - Evolução da Mão-de-Obra por Faixa Salarial - PIM}

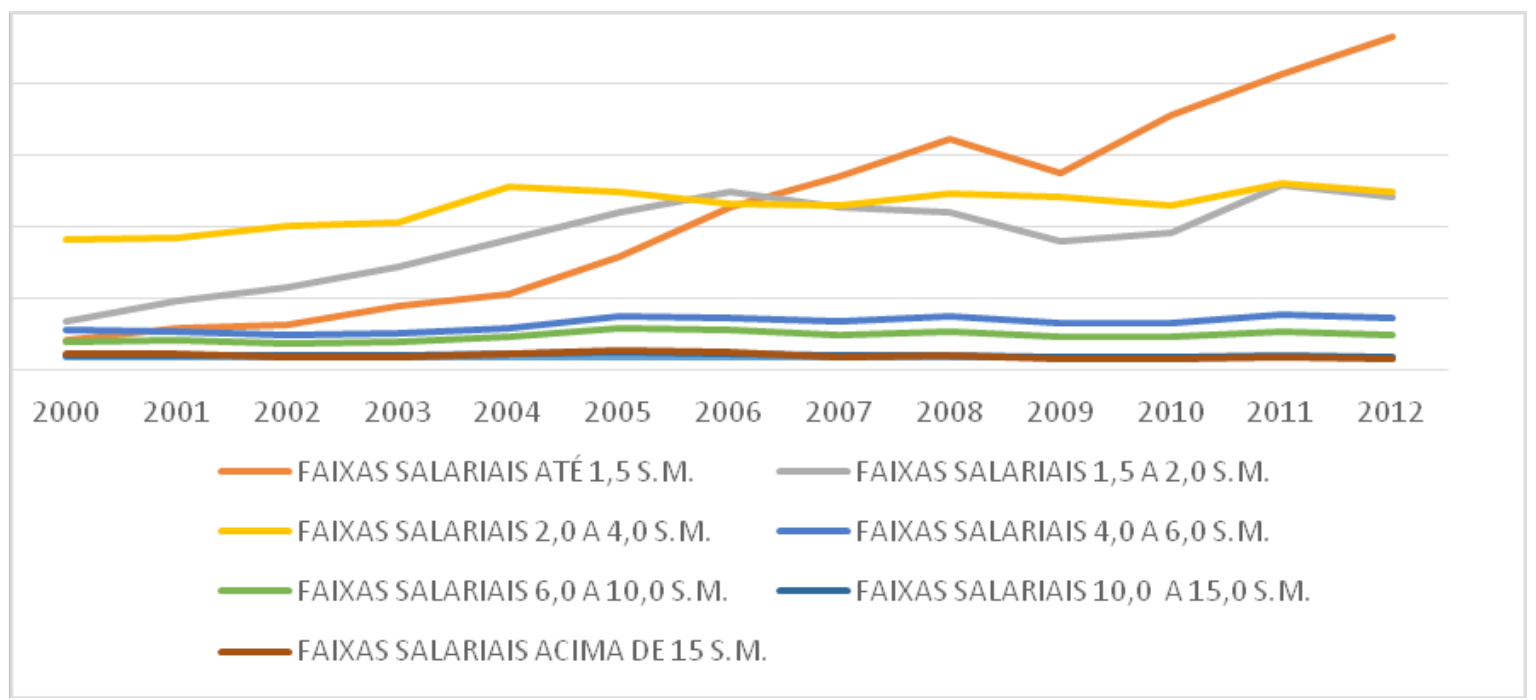

Fonte: Suframa, 2013.

\section{Indicadores de C, T \& I e Rebatimentos Socioeconômicos no Estado do Amazonas}

\subsection{Indicadores do Sistema Regional de Inovação do Estado do Amazonas}

Diniz et at. (2007) calculou um índice de Ciência, Tecnologia e Inovação (ICT\&I) para todos os estados da Amazônia Legal no período de 2000 a 2007. 
A melhor posição no rank dos estados da Amazônia Legal foi ocupada pelo estado do Amazonas, em todo o período considerado ${ }^{3}$. $\mathrm{O}$ incentivo para a dinamização em Ciência e Tecnologia, representado, em especial, para o ano de 2007, está explícito nos índices como: Base Educacional e Disponibilidade de Recursos Humanos Qualificados; Exportação de Produtos Intensivos em Tecnologia; Número de Pesquisadores, Gastos Estaduais com Pesquisa Ciência e Tecnologia, Gastos Federais em fomento a pesquisa, entre outros.

Segundo Diniz (2008), através de pesquisa direta com as empresas do PIM, pode constatar que 68,06\% de suas empresas (numa amostra de 327 empresas) inovam em produtos, especialmente para as unidades situadas em Manaus, além disso, 2,08\% das empresas da amostra atestam que as inovações foram novas para o mercado externo.

Tabela 1 - Comparação da Relação das Empresas Inovadoras com outras Empresas, Governo e Universidades, Centros de Pesquisa entre Brasil e o Estado do Amazonas (Pintec 2003-2005 e 2006-2008)

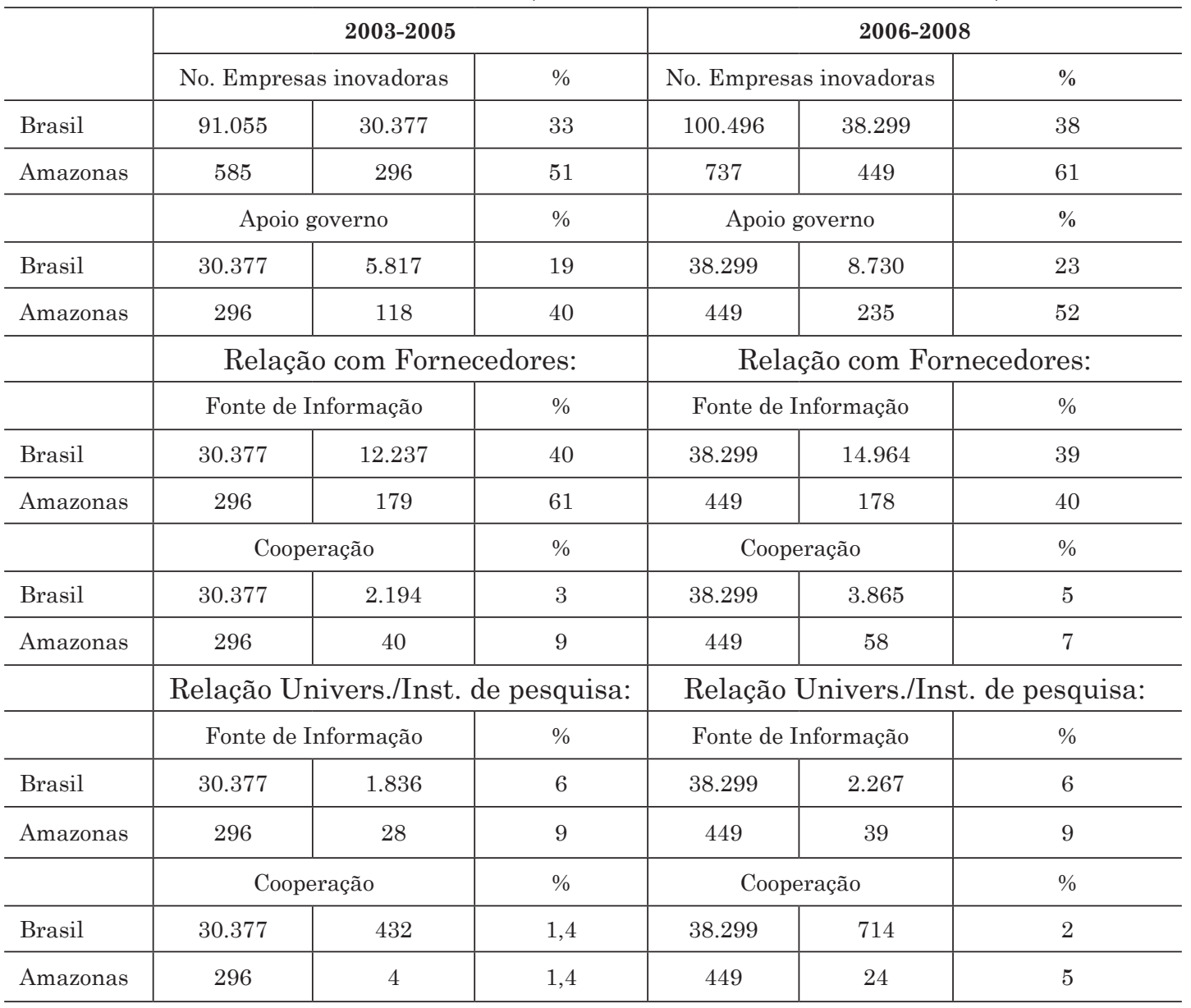

Fonte Pintec 2003-2005 (IBGE, 2006) e Pintec 2006-2008 (IBGE, 2009). Elaboração dos autores.

A posição do estado do Amazonas no índice de ciência, tecnologia e inovação deve-se basicamente ao Pólo Industrial de Manaus. Além disso, seu efeito multiplicador para a economia da cidade de Manaus e para todo o estado é

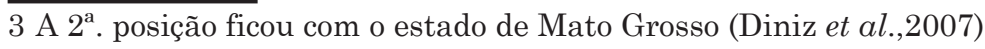


notória, e ainda é o estado que tem os menores índices de desmatamento (Diniz, 2008).

Quando se toma os dados das empresas inovadoras a partir dos dados da PINTEC 2003-2005 (IBGE, 2006) e PINTEC 2006-2008 (IBGE, 2009), o estado do Amazonas apresenta percentualmente um maior número de empresas inovadoras, 33\%, contra 51\%, quando comparado ao Brasil, entre 2003-2005 e, $38 \%$ contra 61\%, respectivamente, entre 2006-2008. Portanto, o estado do Amazonas cresce proporcionalmente mais, comparando os dois períodos.

Por outro lado, na comparação do Estado do Amazonas com o observado para o Brasil como um todo, verifica-se uma maior participação relativa das empresas desse estado em termos do apoio do governo, relação com os fornecedores e relação com universidades e centros de pesquisa que a média nacional, reforçando a ideia de que a tríplice hélice está mais reforçada nesse estado do que no país como um todo.

De fato, as empresas inovadoras do Estado do Amazonas receberam maior apoio do governo que a média nacional, com um crescimento superior entre os dois períodos observados 2003-2005 (193\% contra 40\%) e 2006-2008 (238\% contra 52\%).

A relação com fornecedores quanto à fonte de informações e cooperação é superior para o Estado do Amazonas em comparação ao Brasil, embora tenha decrescido a participação quanto do Estado do Amazonas e crescido para o Brasil entre os dois períodos de análise.

Quanto à importância das Universidades e Centros de Pesquisa, observase uma maior participação do Estado do Amazonas, mantendo-se esta constante quanto à fonte de informação e crescente para o Estado do Amazonas em termos de cooperação entre os dois períodos de análise.

Atestando a interação entre universidades e institutos de pesquisa com as empresas do estado do Amazonas, e assim do PIM, pode-se analisar, através do Censo 2010 do CNPq, o grande número de grupos de pesquisa da Universidade Federal, do Instituto Federal, da Universidade Estadual, da Fundação de Medicina Tropical, da EMBRAPA, todos deste estado, com várias empresas da indústria de transformação do PIM. Em verdade, em aproximadamente $70 \%$ as interações são feitas pelas Universidades Federais e Estaduais, Institutos de Pesquisa Federais e Embrapa.

No gráfico 3 abaixo, pode-se ver o lugar - $2^{\circ}$. lugar- que ocupa o estado do Amazonas em relação aos estados da Amazônia Legal, no que se refere a quantidade de grupos de pesquisa. Posição essa importante quando se leva em conta que quase todas as parcerias feitas são com empresas do Pólo Industrial de Manaus, além de importantes Fundações de Pesquisa privadas como o Instituto Nokia de Tecnologia, entre outros. 


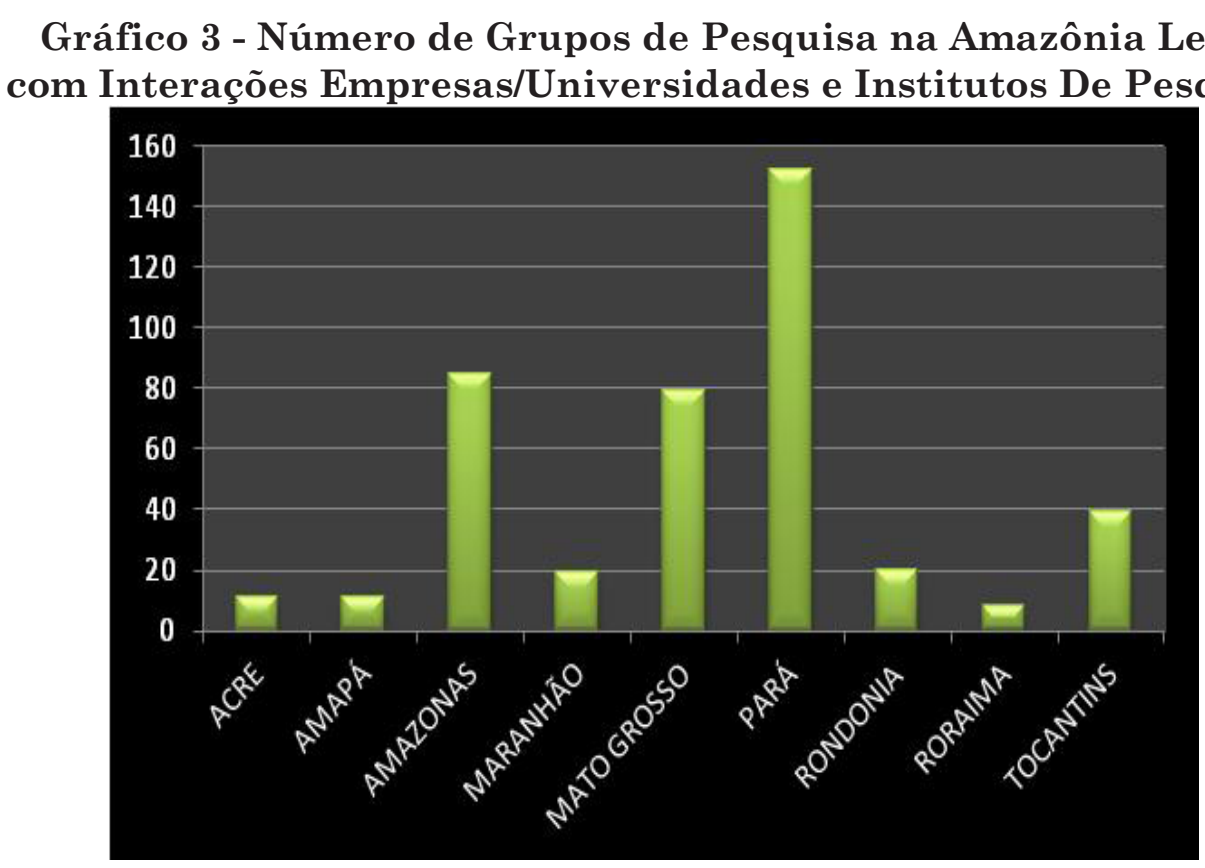

Fonte: Censo CNPq, 2010. http://dgp.cnpq.br/buscaoperacional/

No que se refere à caracterização feita pelo CNPq quanto aos tipos de relacionamentos, vê-se uma predominância do tipo "Pesquisa científica com considerações de uso imediato dos resultados". Além, apesar da menor incidência, do tipo - Transferência de tecnologia desenvolvida pelo grupo para o parceiro; Treinamento de pessoal do parceiro pelo grupo, incluindo cursos e treinamento em serviço; e, Desenvolvimento de software para o parceiro pelo grupo. Pondendo, assim, ser atestado, nesse estado, a interação entre ciência e tecnologia para a atividade de inovação.

A tabela 2 qualifica a relação das empresas inovadoras do Estado do Amazonas e do país como um todo, entre os períodos da Pintec 2003-2005 e Pintec 2006-2008, considerando o tipo de incentivo em incentivo fiscal para P \& D e Lei de Informática, em que se observa mais uma vez a preponderância do Estado do Amazonas. 
Tabela 2 - Percentual da Relação das Empresas Inovadoras com o Governo entre Brasil e o Estado do Amazonas por Tipificação do Incentivo Fiscal (2003-2005 e 2006-2008)

\begin{tabular}{|c|c|c|}
\hline \multicolumn{3}{|c|}{$2003-2005$} \\
\hline & \multicolumn{2}{|c|}{ Tipo de Incentivo } \\
\hline & Incentivo Fiscal & Lei de Informática \\
\hline Brasil & 3,56 & 5,56 \\
\hline Amazonas & 12,96 & 20,99 \\
\hline \multicolumn{3}{|c|}{$2006-2008$} \\
\hline & \multicolumn{2}{|c|}{ Tipo de Incentivo } \\
\hline & Incentivo Fiscal & Lei de Informática \\
\hline Brasil & 5,04 & 8,06 \\
\hline Amazonas & 5,70 & 13,17 \\
\hline
\end{tabular}

Fonte Pintec 2003-2005 (IBGE, 2006) e Pintec 2006-2008 (IBGE, 2009). Elaboração dos autores.

Os rebatimentos que esses indicadores de inovação, acima discutidos, impõem ao estado do Amazonas são evidenciados por alguns indicadores econômicos e sociais. Conforme dados do IBGE (2010) o estado do Amazonas possui o maior IDH $(0,780)$ e o maior o PIB per capita entre todos os estados do Norte do Brasil. Como era de se esperar, o setor secundário é o que apresenta maior importância no papel econômico do estado em função do Polo Industrial de Manaus, e é o segundo maior centro de indústria do Brasil. O setor terciário aparece em segundo lugar.

\section{Conclusão}

O Sistema Nacional de Inovação brasileiro pode ser situado em um nível intermediário de construção, segundo Suzigan e Albuquerque (2011). Para essa afirmação, esses autores elucidam que existem alguns critérios para classificação desse Sistema como imaturo, intermediário ou desenvolvido. Desses, dois critérios podem ser considerados nesse artigo. O primeiro referese à existência de instituições de pesquisa e ensino construídas, mobilizando pesquisadores, cientistas e engenheiros com o objetivo de dinamizar, através da inovação, o sistema produtivo. O segundo é identificado como o envolvimento das firmas com um processo de inovação contínuo.

O diagnóstico do Brasil, para tanto, é a existência em todo o território nacional de "pontos de interação" (locais ou estaduais) formados por relações mais fortes entre universidades e empresas, além do apoio governamental em suas várias instâncias - hélice tripla.

Dessa forma, pode-se considerar, através da própria construção histórica feita para o Polo Industrial de Manaus e os diversos indicadores econômicos e, em especial os indicadores sobre inovação do estado do Amazonas (PINTEC/ IBGE), esse estado como um "ponto de interação" no cenário nacional.

Em verdade, a dinâmica do Polo Industrial de Manaus funciona como um circuito retroalimentador positivo para o estado do Amazonas, inclusive com efeitos sociais positivos, conformando, assim, um Sistema Regional/Local 
de Inovação intermediário, ou até mesmo, desenvolvido em relação a alguns estados brasileiros.

\section{Referências}

Albuquerque, E. M.; Silva, L. A.; Povoa, L. (2005). Diferenciação Intersetorial na Interação entre Empresas e Universidades no Brasil: Notas Introdutórias sobre as Especificidades da Interação entre Ciência e Tecnologia em Sistemas de Inovação Imaturos. Texto para Discussão $\mathrm{N}^{\circ}$ 264. Belo Horizonte: UFMG/ CEDEPLAR.

Asheim, B./ Vang, J. (2004). What can regional systems of innovation and learning regions offer developing regions? Beijing. (Paper presented at the second Globelics conference in Beijing, October 2004).

Becker, Bertha K. (2006). Da preservação à utilização consciente da biodiversidade Amazônica. O papel da ciência, tecnologia e inovação. Em: Irene Garay; e Bertha K. Becker (org.). "As dimensões humanas da Biodiversidade. O desafio de novas relações na sociedade no sec. XXI.” Petrópolis: Vozes.

Becker, B K. (1985). Fronteira e urbanização repensadas. Revista Brasileira de Geografia, 47(3/4), 357-371.

Becker, Bertha K. (2005). Geopolítica Da Amazônia. Estudos Avançados, 19, 71-86.

Becker, Bertha Koiffmann, De Assis Costa, F., Da Costa, W. M., De Oliveira, A. U., Yared, J. A. G., Benatti, J. H., De Miranda, M. H. S. P., et al. (2009). "Um projeto para a Amazônia no século 21: desafios e contribuições.” (A. C. F. Galvão, B. K. Becker, F. de Assis Costa, \& W. M. da Costa, Eds.) (p. 428). Brasilia - DF: Centro de Gestão e Estudos Estratégicos - CGEE.

Brisolla, Sandra; Corder, Solange; Gomes, Erasmo; Mello, Débora. (1997). As relações universidade-empresa-governo: um estudo sobre a Universidade Estadual de Campinas. Educação \& Sociedade, ano XVIII, nº 61, Dezembro. p. 187 - 208.

Cano, W. (1985). "Desequilíbrios Regionais e Concentração Industrial no Brasil." São Paulo:Global (PNPE,Teses, 15).

Cano, Wilson. (1997). Concentração e Desconcentração Econômica e Regional no Brasil: 1970/95. Economia e Sociedade 8. Campinas: Junho.

Cassiolato, J. E; Lastres, H. M. M. (1998). Local Systems of Innovation in the Mercosur of the 1990s: A Contribution to the Debate on S\&T Policy Decentralization. Paper apresentado no Workshop "Techregiões: ciência, tecnologia e desenvolvimento - passado, presente e futuro", realizado no Rio de Janeiro.

Cassiolato, José Eduardo (coord.) (2010). Perspectivas do investimento na economia do conhecimento. Rio de Janeiro: Synergia: UFRJ, Instituto de Economia; Campinas: UNICAMP, Instituto de Economia. (Projeto PIB - Perspectivas investimento no Brasil, v. 3).

Cassiolato, J. E.; Lastres, H. M. (2008). Discussing innovation and development: converging points between the Latin American scholl and the innovation Systems perspective? GLOBELICS. 
Cohen, W. M.; Nelson, R. R.; Walsh, J. P. (2002). The Influence of Public Research on Industrial R\&D. Management Science, v. 48, n.1, p.1-23, Jan.

Campanário, M. de A. (2002). Tecnologia, Inovação e Sociedade. Trabalho apresentado no seminário VI Módulo de la Cátedra CTS I Colombia, llamado "Innovación Tecnológica, Economia y Sociedad".

Campos, I. M.; Valadares, E. (2008). Inovação Tecnológica e Desenvolvimento Econômico.

Cohen, Wesley, M. \& Levinthal, D. A. (1990). "Absorptive Capacity: A New Perspective on Learning and Innovation”, ASQ, 35, 128-152.

Cooke, P. (2001). Regional innovation systems clusters and the knowledge economy. Oxford Journals Economics \& Social Sciences Industrial and Corporate Change. Volume 10, Issue 4, Pp 945-974.

Coutinho, L. G.(1994). "Superação da fragilidade tecnológica e a ausência de cooperação. Ciência e tecnologia: alicerces do desenvolvimento." São Paulo: Cobram, p.119.

Coutinho, L; Ferraz, J. C. (1994). Estudo da competitividade da indústria brasileira. Campinas: Papirus; Unicamp.

Costa, F. de A. (1998). "Ciência, tecnologia e sociedade na Amazônia: questões para o desenvolvimento sustentável." Belém: Cejup.

Dagnino, Roberto (2003). A relação Universidade-Empresa no Brasil e o Argumento da Hélice Tripla. Revista Brasileira de Inovação; Volume 2; Número 2 ;Julho / Dezembro.

Delgado, D. M. (2006). Inovação, Tecnologia e Desenvolvimento Econômico: a universidade como locus privilegiado das demandas empresariais. In: Reunião Anual da ANPED - Associação Nacional de Pós-Graduação e Pesquisa em Educação, 29., Caxambu. Anais Eletrônicos.

De Negri, J.A.; Salerno, M. S., (2005) (Org). Inovações, padrões tecnológicos e desempenho das firmas industriais brasileiras. Brasília: IPEA.

Diniz, C. C. (1995). A Dinâmica Regional Recente da Economia Brasileira e suas Perspectivas. Texto para Discussão no. 375. IPEA, Junho.

Diniz, M. B.; Santos, R. B. N.; Diniz, M. J. T.; Puty, C.; C. B.; Rivero, S. L. de M. (2007). A Amazônia (Legal) Brasileira: evidências de uma condição de armadilha da pobreza?. $35^{\circ}$ Encontro Nacional de Economia. Anais. Recife-Pe.

Diniz, M. J. T. (2008). A Dinâmica das inovações nas empresas do pólo industrial de Manaus: um novo momento relacionado aos constrangimentos ambientais a partir do ano de 2000. Tese (Doutorado) - Universidade Federal do Pará, Núcleo de Altos Estudos Amazônicos, Belém,

Diniz, M.J.T.; Almeida, L.M.; Diniz,M.B.; Santos, M.S.X. (2009). Evolução dos Índices de Ciência, Tecnologia e Inovação - ICT\&I- para os Estados da Amazônia Legal no Período entre 2000 e 2007. Novos Cadernos do NAEA, Belém/PA.

Doloreux, D.; Hommen, D.. (2003). Is the Regional Innovation System Concept at the End of Its Life Cycle? Paper presented for the conference Innovation in Europe: Dynamics, Institutions and Values, Roskilde University, Denmark. 
Dosi, G. (2006). "Mudança Técnica e Transformação Industrial. Um Estudo da Industria de Semicondutores." São Paulo: Unicamp.

Dosi, G. (1984). Technology and Conditions of Macroeconomic Development. In: Freeman, C. (Ed.). "Design, Innovation and Long Cycles in Economic Development.” New York: St. Martin's Press.

Dosi, G.; Freeman, C.; Nelson, R.; Silverberg, G.; Soete, L. (Ed.). “Technical Ceange and Economic Theory." London: Pinter, 1988.

Edquist, C. (1997). "Systems of Innovation - Technologies, Institutions and Organizations", London: Pinter.

Edquist, C. (2009). Systems of innovation. Perspectives and Challenges. Em: J. Fagerberg; Ehrlich P. H.; Ehrlich, A. “The dominant animal.” Island Press.

Etzkowitz, H.; Leydesdorff Loet. The Triple Helix as a Model for Innovation Studies (Conference Report), Science\& Public Policy Vol. 25(3) (1997) 195-203

Etzkowitz, H., And Loet Leydesdorff (EDS.) (1997). "Universities and the Global Knowledge Economy: A Triple Helix of University-Industry-Government Relations." London: Cassell Academic.

Etzkowitz, H., And Loet Leydesdorff (1998). “The Endless Transition: A 'Triple Helix' of University-Industry-Government Relations," Minerva (Fall).

Etzkowitz H; Webster,A; Gebhardt C; Terra,B. (2000). The future of the university and the university of the future: evolution of ivory tower to entrepreneurial paradigm. Research Policy, Volume 29, Issue 2, February, Pages 313-330

Fagerberg, J. (2005). Innovation: a guide to the literature. Em: J. Fagerberg; D. Mowery; R. Nelson R. (eds). “The Oxford Handbook of Innovation.” New York, USA: Oxford University Press, p. 1-26.

Farinha, L.; Ferreira, J.J. (2013). TripleHelix Association. Working Paper (WP). London/Ingland.

Freeman, C. (1995). The National System of Innovation in historical perspective. Cambridge Journal of Economics, V.19, n.1. P.5-24.

Freeman, C.; Soete, L. (2008). “A Economia da Inovação Industrial”. São Paulo: Unicamp.

Galbraith, J. K. (1988). “O Novo Estado Industrial.” São Paulo: Nova Cultural.

Garcia, E. (2004). "Zona Franca de Manaus: história, conquistas e desafios.” Manaus: Norma/SUFRAMA.

Inzelt, A. (2004): The evolution of university-industry government relationships during transition. Research Policy, (33)6/7, 975-995.

Klevorick, A. K.; Levin, R.; Nelson, R.; Winter, S. (1995). On the Sources and Significance of Interindustry Differences in Technological Opportunities. Research Policy, v.24, n.2, p.185-205, Mar..

Lundvall, B. (ed.) (1992). "National Systems of Innovation: toward a theory of innovation and interactive learning." London: Pinter.

Lundvall, B. (2003). National Innovation Systems: history and theory. In Elgar "Companion to Neo-Schumpeterian Economics", Cheltenham: Edward Elgar. 
Machado, J. A. da C. (2006). et al. Metamorfoses do modelo Zona Franca de Manaus: desafios à pesquisa e ao planejamento do desenvolvimento regional. In: Scherer, Elenise; Oliveira, José Aldemir de. (Orgs.). "Amazônia: políticas públicas e diversidade cultural.” Rio de Janeiro: Garamond. 260 p; 16:23. (Terra Mater).

Martinello, P. A (1988). Batalha da Borracha durante a Segunda Guerra Mundial. Rio Branco: Cadernos UFAC, série Estudos e Pesquisa 1.

Mytelka. L; Farinelli. F (2005). De Aglomerados Locais a Sistema de Inovação. Em José Eduardo Cassiolato; Helena Lastres; Ana Arroio (org.). "Conhecimento, sistemas de inovação e desenvolvimento.” Rio de Janeiro: Editora UFRJ; Contraponto.

Nelson, R. R.; Rosenberg, N. (1993). Technical innovation and national systems. In: Nelson, R.(ed). "National innovation systems: a comparative analysis." New York, Oxford: Oxford University.

Nelson, R. R.; Winter, S.G. (2005). Uma Teoria Evolucionária da Mudança Econômica. São Paulo: Unicamp.

Oreiro, J. L. (1999). Progresso Tecnológico, Crescimento Econômico e as Diferenças Internacionais nas Taxas de Crescimento da Renda per capita. Uma Crítica aos Modelos Neoclássicos de Crescimento. Economia e Sociedade.

Rapini, M. (2004). Interação Universidade - Indústria no Brasil: Uma Análise Exploratória a Partir do Diretório dos Grupos de Pesquisas do CNPq. Rio de Janeiro, Instituto de Economia, Universidade Federal do Rio de Janeiro.

Rapini, M. S.; Righi, H. M. (2005). Interação Universidade-Empresa no Brasil em 2002 e 2004: Uma Aproximação a partir dos Grupos de Pesquisa do CNPq, Maio.

Rapini, M. S.; Righi, H. Metodologia para o Mapeamento da Interação UniversidadeEmpresa a partir do Diretório dos Grupos de Pesquisa do CNPq no Censo 2004, Versão de Julho de 2006.

Righi, H. M. (2005). Interação Universidade-Empresa em Minas Gerais: Uma Análise Exploratória a partir do Diretório de Grupos de Pesquisa do CNPq, Belo Horizonte.

Rivas, Alexandre; Mota José Aroudo; Machado José Alberto (coord.) (2008). Como Proteger A Amazônia? Discurso Ou Fato: Evidências Do Pólo Industrial De Manaus.Manaus-Am, Instituto Piatam.. Relatório de pesquisa, Convênio Instituto Piatam, Suframa, Nokia (versão 7.)

Rocha, E. M. P. E Ferreira, M. A. T. (2004). Indicadores de Ciência, Tecnologia e Inovação: mensuração dos Sistemas de CT\&I nos Estados Brasileiros. Ciência da Informação, Vol. 33, no.3.

Secretaria De Estado De Planejamento E Desenvolvimento Econômico - SEPLAN/ Governo do Estado do Amazonas. Balança Comercial do Estado do Amazonas em 2012. Amazonas-Manaus, 2013

Secretaria De Estado De Planejamento E Desenvolvimento Econômico - SEPLAN/ Governo do Estado do Amazonas. Balança Comercial do Estado do Amazonas em 2012. Amazonas-Manaus, 2013

Superintendencia Da Zona Franca De Manaus (SUFRAMA) . Indicadores do PIM 2008 a 2013; 1988 a 2010. 
Superintendencia Da Zona Franca De Manaus - SUFRAMA. Relatório Anexo - Gestão 2006.

Suzigan, W. E Albuquerque, E. M. (2006) Projeto de Pesquisa: Interações de Universidades e Institutos de Pesquisa com Empresas no Brasil.

Suzigan, W. E Albuquerque, E. M.; Cário, S. (2011). "Em busaca da Inovação: Interação Universidade-Empresa no Brasil.” Wilson Suzigan, Eduardo da Motta e Albuquerque, Silvio Antonio Ferraz Cário (Org). Belo Horizonte: Autentica Editora.

Villaschi, A. (1996) "Paradigmas e Desenvolvimento, Oportunidades e Desafios para a Economia Brasileira.” EDUFES,

Webster, A.J.; Etzkowitz, H., (1991). "Academic-industry relations: the second academic revolution?”, 31 p.,London: Science Policy Support Group. (SPSG concept paper n.12).

Winter, S. (1984). "Schumpeterian Competition in Alternative Technological Regimes", p.287-320.

Sites Consultados :

www.suframa.gov.br

www.seplan.am.gov.br

www.cnpq.br

www.ibge.gov.br

www.pintec.ibge.gov.br 
